\title{
The summing up
}

\author{
Sir Arnold Burgen (National Institute \\ for Medical Research, Mill Hill, \\ London, UK)
}

Receptors in the sense used in this symposium fall into two classes, namely those on the outer surface of the membrane whose message must be transmitted to the cell interior and those already in the interior. The first of these classes is embedded in a membrane and is constrained to act in two dimensions, and it is all too tempting to regard the lipid phase of the membrane as a passive supporting matrix, yet the effects on phospholipid turnover described in this symposium point not only to a more dynamic role for the lipid, but perhaps even to a metabolic imperative occurring in the relatively anhydrous environment of the lipid leaflet. Yet there are events at the motor end-plate and in the sino-auricular node which seem free of this requirement and are concerned with coupling between receptor and ionophoric channels.

The more common process seems to be one in which the receptor is coupled to an enzyme orientated to the inner face of the membrane, the best understood of these being adenylate cyclase. The evidence accumulates that this may be a fairly complex assembly including modulatory subunits and faces us with the confusion of terminology because the term receptor can be applied to a binding subunit or to the complex assembly which includes the effector. The analysis of these complexes is challenging and requires both methods of purification and assay methods involving reconstitution in incomplete systems such as the Friend erythroleukaemia cell described in this symposium. An alternative strategy is to use monoclonal antibodies raised against the receptor complex to identify the number of functional units.

When the messenger is released inside the cell the consequences are usually multiple even though the message is rather simple: Ca or cyclic AMP for instance. The real complications of intracellular messages are seen with the steroid receptor which doubles also as a message.

This symposium records some of the astonishing growth of understanding concerning receptors and their effectors that has occurred in recent years; it records many unanswered questions, but the considerable activity in this field assures us that many will be answered before long. 